\title{
The Simpson grade: abandon the scale but preserve the message
}

\author{
Theodore H. Schwartz, MD, ${ }^{1}$ and Michael W. McDermott, MD² \\ 1Department of Neurosurgery, Otolaryngology and Neuroscience, Weill Cornell Medicine, NewYork-Presbyterian Hospital, \\ New York, New York; and 'Division of Neuroscience, Translational Medicine, Herbert Wertheim College of Medicine, Florida \\ International University, Miami, Florida
}

\begin{abstract}
The Simpson grading scale, developed in 1957 by Donald Simpson, has been considered the gold standard for defining the surgical extent of resection for WHO grade I meningiomas. Since its introduction, the scale and its modifications have generated enormous controversy. The Simpson grade is based on an intraoperative visual assessment of resection, which is subjective and notoriously inaccurate. The majority of studies in which the grading system was used were performed before routine postoperative MRI surveillance was employed, rendering assessments of extent of resection and the definition of recurrence inconsistent. The infiltration and proliferation potential of tumor components such as hyperostotic bone and dural tail vary widely based on tumor location, as does the molecular biology of the tumor, rendering a universal scale for all meningiomas unfeasible. While extent of resection is clearly important at reducing recurrence rates, achieving the highest Simpson grade resection should not always be the goal of surgery.

Donald Simpson's name and his scale deserve to be recognized and preserved in the historical pantheon of pioneering and transformative neurosurgical concepts. Nevertheless, his eponymous scale is no longer relevant in modern meningioma surgery. While his message of maximizing extent of resection and minimizing morbidity is still germane, a single measure using subjective criteria cannot be applied universally to all meningiomas, regardless of location. Meningioma surgery should be performed with the goal of achieving maximal safe resection, ideally guided by molecularly tagged fluorescent labeling and assessed using objective criteria, including postoperative MRI as well as molecularly tagged scans such as [68Ga]-DOTATATE-PET.
\end{abstract}

https://thejns.org/doi/abs/10.3171/2020.6.JNS201904

KEYWORDS dural tail; extent of resection; hyperostosis; meningioma; recurrence; surgery; Simpson grade; skull base; oncology

$\mathrm{T}$ HE Simpson grading system, introduced in 1957 by Donald Simpson in his landmark paper "The recurrence of intracranial meningiomas after surgical treatment," has been the gold standard in defining the surgical objectives of meningioma surgery. ${ }^{1}$ Simpson presumed that since meningiomas were benign tumors, there would be a direct correlation between the degree of resection and the rate of recurrence. Since its publication, his 5-point scale has generated a great deal of controversy regarding the accuracy of its predictions. Meningiomas have more recently been divided into biological classes, most notably by the WHO grading system. ${ }^{2}$ Simpson's scale is now only applicable to WHO grade I meningiomas, and, even with this histological limitation, its accuracy and value remain a question of ongoing debate. This article will review the literature on the Simpson grade and attempt a critical analysis of the evolution in thought regarding this historically important but subjective scale.

\section{Historical Context}

Simpson introduced his grading system in response to the observation that recurrences frequently occurred even after seemingly complete meningioma resections. Simpson created a grading scale to predict the incidence of recurrences based on the quantifiable metrics of the day and his understanding of the biology of the tumor. He explained, "It is more profitable to consider these recurrences as the expression of a failure in surgery, a failure to eradicate microscopic infiltration...." Simpson's 5-point scale (Table 1) is based on the assumptions that 1) the surgeon's intraoperative macroscopic inspection could be used to determine complete tumor removal; 2) the dural attachment, tumor

ABBREVIATIONS GTR = gross-total resection; STR = subtotal resection .

SUBMITTED May 20, 2020. ACCEPTED June 16, 2020.

INCLUDE WHEN CITING Published online October 9, 2020; DOI: 10.3171/2020.6.JNS201904. 
TABLE 1. The Simpson scale

\begin{tabular}{cl}
\hline Grade & \multicolumn{1}{c}{ Definition } \\
\hline I & $\begin{array}{c}\text { Macroscopic complete tumor resection with removal of } \\
\text { affected dura and bone }\end{array}$ \\
\hline I & $\begin{array}{c}\text { Macroscopic complete tumor resection with coagulation of } \\
\text { affected dura }\end{array}$ \\
\hline III & Macroscopic complete tumor resection \\
\hline IV & Subtotal tumor resection \\
\hline V & Decompression with/without biopsy \\
\hline
\end{tabular}

Based on information provided in Simpson D. The recurrence of intracranial meningiomas after surgical treatment. J Neurol Neurosurg Psychiatry. $1957 ; 20(1): 22-39.1$

invading an adjacent sinus, and the nearby hyperostotic bone possessed an equal potential for tumor regrowth; 3 ) coagulation of the dural attachment could reduce the rate of regrowth, but not to the same extent as complete removal; and 4) the size of the residual tumor was not a factor in predicting recurrence. ${ }^{1}$

Simpson himself recognized the limitations of his own scale and the subjectivity of relying on intraoperative assessments. He noted, "it is not possible to determine from his $\left[\mathrm{Grant}^{49}\right]$ account whether these 'incomplete removals' had been recognized as such initially, or whether they had appeared complete resections at the original operation." Later in the paper, he explicitly confirmed, "It is of course true that the surgeon's assessment is not always correct, especially with the deeper tumours." In reading Simpson's original article, it is apparent that the grades were derived from a retrospective review of operative reports, many of which were not even dictated by the surgeons themselves. He also recognized the inadequacy of grade IV and its broad category of subtotal resection (STR): "The operations ranged widely in magnitude, from those in which the residue was estimated as 'only a thin shell' or ... to those in which at least half the tumour was left in situ."

Simpson also expressed ambivalence about the importance of hyperostotic bone. He wrote, "Recurrence from invaded bone, over which Cushing (1938) appears to have been much concerned, seems unusual ... proof is lacking." $1,3 \mathrm{He}$ continued, "Invasion of bone cannot be ignored, but it appears of overrated importance: when one recalls how frequently meningiomas are associated with hyperostosis or bony erosion, it is surprising how rare is a recurrence clearly arising from bone." Finally, absent any method for reliable postoperative imaging, Simpson had to rely on the return of clinical symptoms. "The term 'recurrence' is used in a purely clinical sense to imply the reappearance of symptoms due directly to tumour growth after a period of symptomatic relief."

\section{Evidence Against the Accuracy of the Simpson Grade}

Following Simpson's seminal publication, several articles were published examining the relationship between extent of resection and recurrence in meningiomas. One of
TABLE 2. Limitations to the Simpson grade and the supporting research studies

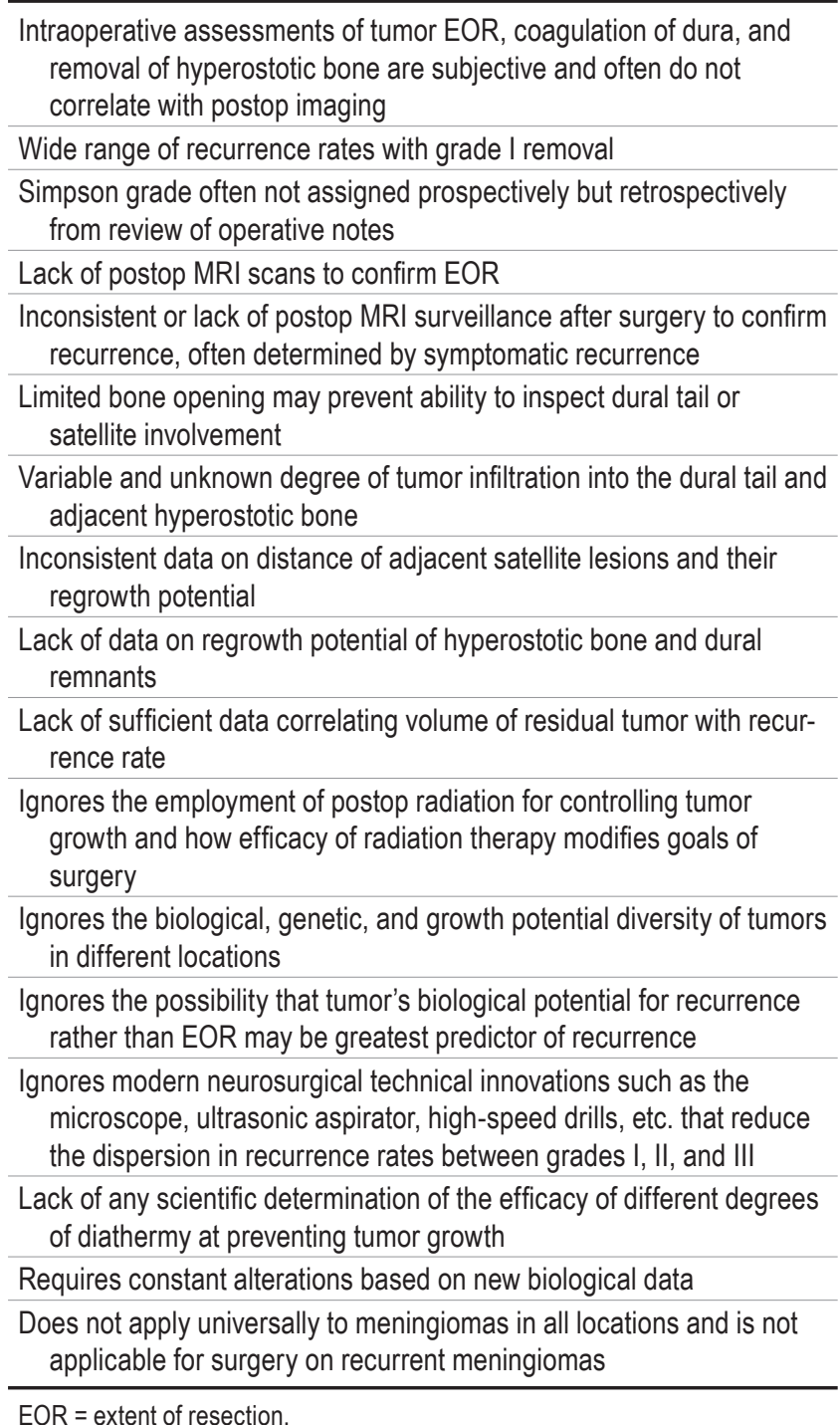

EOR $=$ extent of resection.

the most common findings was a confirmation that extent of resection was predictive of recurrence, but the methodology employed in these studies did not support Simpson's actual scale. Adegbite et al., ${ }^{4}$ Mirimanoff et al., ${ }^{5}$ and Naumann and Meixensberger ${ }^{6}$ published articles between 1980 and 1990 linking extent of resection with recurrence, but these studies were only able to find significance by dividing resections into two categories, namely, surgeon's subjective intraoperative visual estimate of gross-total resection (GTR) versus STR. Wide variability in tumor recurrence after a supposed Simpson grade I resection $(9 \%-55 \%)$ was a clear indication of the limitations and subjectivity of the scale. ${ }^{1,4,5,7}$ These early studies were also limited by the same problems faced by Simpson, namely, a lack of adequate microscopic technique, unreliable estimates of extent of resection gathered from retrospective operative report review, and an inability to detect asymptomatic recurrences (Table 2). 

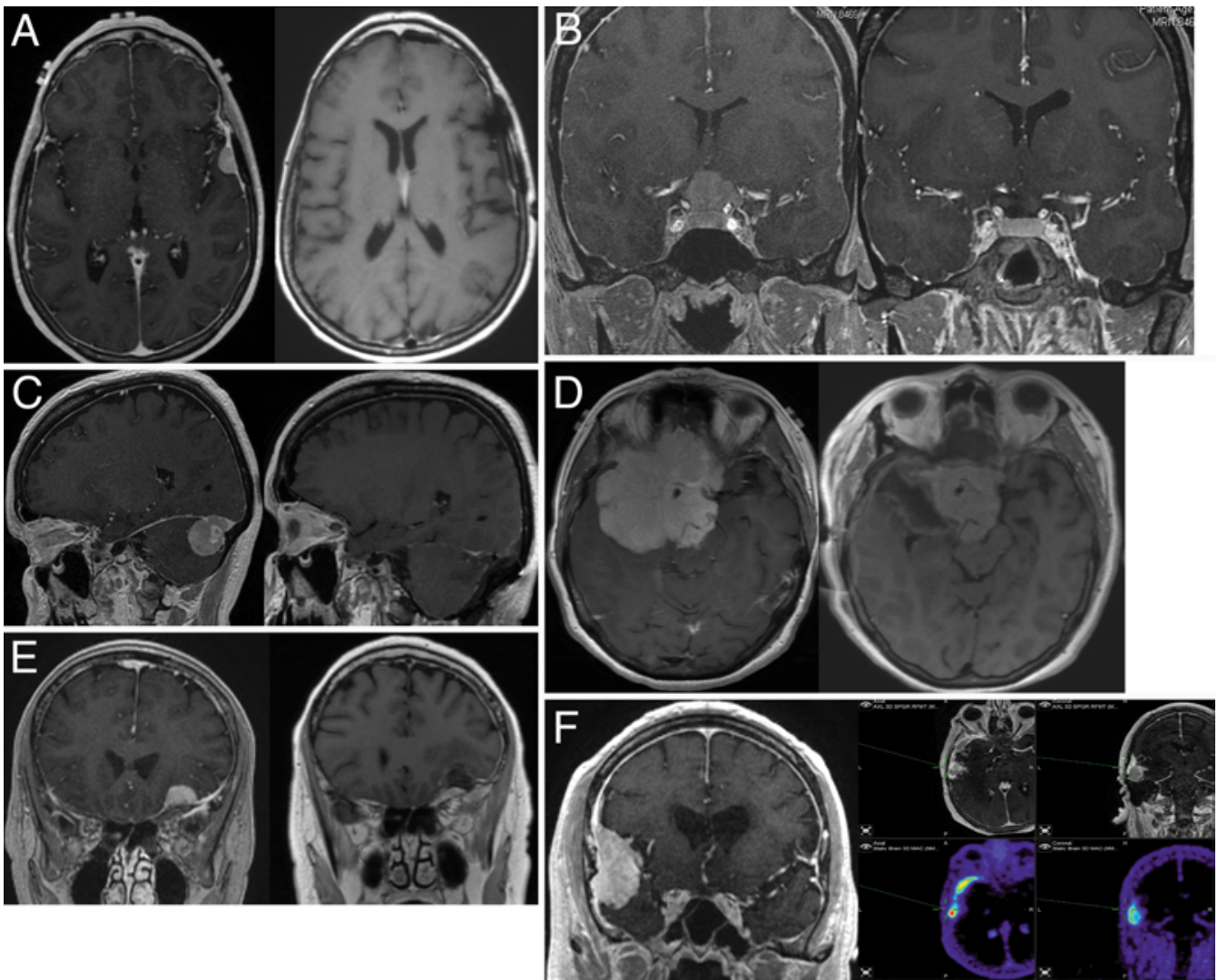

FIG. 1. Limitations of the Simpson grading scale. A: Axial images of a grade I resection of a small convexity meningioma. Whether the bone opening was sufficiently large to remove or even if the extent of lateral dural attachment could be observed is unknown. Should a $10-\mathrm{cm}$ craniotomy be performed in this case to ensure a grade 0 removal? B: Coronal images of another grade I resection performed through an endonasal approach of this tuberculum meningioma. Is the recurrence rate for this skull base meningioma the same as that for the small convexity meningioma in panel $\mathrm{A}$ ? Can the dural tail be adequately visualized through this approach to even assign a reliable Simpson grade? C: Sagittal images showing Simpson grade IV removal in which the surgeon left a small remnant invading the transverse sinus. D: Axial images of another Simpson grade IV removal in which the surgeon left tumor encasing the internal carotid, middle cerebral, posterior cerebral, and basilar arteries. Is the recurrence rate in panel $D$ the same as in panel C? E: Coronal images showing a Simpson grade II removal in which hyperostosis of the orbital roof was partially removed. Is the potential for regrowth the same as if no hyperostosis were removed or if the dural tail were not coagulated? F: Lateral convexity meningioma with a dural tail. How much of the dural tail is tumor versus increased vascularity? If the bone opening fails to reveal the extent of dural involvement but the surgeon is not aware, as in panel B, might the grade assigned be inaccurate? The panel on the right shows intraoperative stereotactic coregistered [68Ga]-DOTATATE-PET to determine the extent of tumor in the dural tail.

\section{Simpson Grade 0 and the Concept of Regional Multifocality}

Two additional back-to-back seminal publications by Borovich and Doron seemed to provide an explanation for the high recurrence rates found in earlier studies, namely, the existence of small macroscopic and even microscopic satellite clusters of meningiomatous satellites found as far away as $4 \mathrm{~cm}$ from the edge of the main tumor. ${ }^{8}$ Such "regional multifocality" was not a rare phenomenon and occurred in as many as $67 \%$ of samples. ${ }^{9}$ In order to salvage the Simpson grading scale, Borovich et al. introduced the idea of a grade 0 resection that would include $4 \mathrm{~cm}$ of surrounding dura and thereby preserve Simpson's goal of a surgical cure for meningiomas. ${ }^{10}$ In support of this hypothesis, Kinjo et al. performed grade 0 resections in a series of convexity meningiomas and reported no recurrences after 5 years. ${ }^{11}$
The implication of these articles was that Simpson was basically correct in his scale and its assumptions but that the criteria for grade I were not adequately stringent. However, viewed in another light, these articles raise doubt about the very concept of defining a surgical goal that will cure all meningiomas. First, the very fact that the highest Simpson grade can be modified based on new biological data means that one must be willing to constantly upgrade the Simpson grading scale with every new discovery. Eventually, the Simpson grade will evolve to look nothing like the original scale. Moreover, if regional multifocality occurs in convexity meningiomas, there is no reason why it would not occur in all meningiomas, regardless of location. If this is the case, then achieving a Simpson grade 0 is a near impossibility, except in the rare case of a small isolated convexity meningioma (Fig. 1).

In another series of convexity meningiomas, Moro- 
koff et al. reported a 1.8\% 5-year recurrence rate with resection of a mere 5-mm margin of dura, and these few recurrences had borderline atypical histology on reoperation..$^{12}$ Although ostensibly written in support of Simpson grade I resection, the paper raised two other criticisms of the Simpson scale: first, that residual meningiomatous satellites might not always have the same potential for regrowth since only $5 \mathrm{~mm}$ of surrounding dura was removed, and second, that histological progression might be the greatest predictor of recurrence regardless of extent of resection. ${ }^{13}$

\section{Simpson Grades I-III}

Starting in 2010 with the publication of Sughrue et al., several papers emerged openly that questioned the modern-day relevance of the Simpson scale and demonstrated little difference in recurrence rates for Simpson grades I, II, III, and even IV.14 The authors mainly attributed the breakdown of the Simpson grade to the evolution of modern microneurosurgical technique and the small amount of residual tumor remaining even after STR in combination with devascularization from embolization. Oya et al. likewise reported no difference in recurrence for WHO grade I, II, and III tumors. ${ }^{15}$ A similar report by Otero-Rodriguez et al. found no difference in recurrences between grade I, II, and III tumors but again relied on retrospective review of operative notes to determine the Simpson grade and did not use postoperative MRI scans to validate their determinations. ${ }^{16}$ On the other hand, a study by Ehresman et al., which used postoperative MRI scans rather than the surgeon's intraoperative assessment to determine the Simpson grade, reported no correlation between Simpson grade and recurrence. ${ }^{17}$

Tumor location has also been investigated as a critical factor in the utility of the Simpson grading system. Voß et al. reported that, while extent of resection generally predicted recurrence for all locations, significance was only achieved for Simpson grade IV resections in convexity and skull base tumors. ${ }^{18}$ For tumors in all other locations, neither Simpson grade nor extent of resection was predictive. One of the strengths of that study was that Simpson grading was performed by the surgeon in a prospective manner. This paper again raised the question of the Simpson scale's universality. If the scale is only accurate in certain locations, then it loses its value.

\section{Simpson Grade IV}

Another weakness of the Simpson grading system is the grade IV category, which includes all patients with STR. Simpson did not to take into account that the volume of residual tumor might impact recurrence, likely because postoperative CT and MRI scans were not available to provide such a measurement (Fig. 1). Fukushima et al. examined a series of grade IV meningiomas and showed that a volume of residual tumor $>4 \mathrm{~cm}^{3}$ predicted recurrence on univariate analysis. ${ }^{19}$ Materi et al. posed a similar question and used postoperative volumetric MRI to examine patients with Simpson grade IV resections. ${ }^{20}$ The authors reported that residual volume $>5 \mathrm{~cm}^{3}$ was associated with increased growth rate.

\section{Radiation}

Another criticism of the Simpson scale emerged from the ever-increasing use of stereotactic radiosurgery to manage asymptomatic radiographic recurrences. Described by Black et al., ${ }^{21}$ the philosophy of conservative surgery for skull base meningiomas to restore and preserve function relied on postoperative radiation therapy to manage residual tumor growth. Likewise, McGovern et al. reported that progression-free survival was similar for meningiomas treated with GTR compared with those treated with STR plus radiation. ${ }^{13}$ The authors also showed the difficulty in assigning a Simpson grade using a retrospective review of the surgeon's operative notes. These authors recommended merely classifying surgeries as either GTR or STR as the most reliable predictive scale. A similar conclusion was reached by Condra et al., who found no difference in progression rates when comparing Simpson grade I, II, and III resections and no difference between GTR and STR plus radiation therapy. ${ }^{22}$

In these articles, the increasing use of radiation therapy can be viewed from one perspective as a shift in the philosophical goal of meningioma surgery away from GTR toward safe STR. However, viewed from another perspective, GTR can still be the goal of meningioma surgery, but the threshold and definition of "safe" is lowered. Given the efficacy of postoperative SRS to small residual tumors, tolerating a functionally important neurological deficit to achieve GTR no longer makes sense. Nevertheless, if GTR can be achieved with acceptably low morbidity, it should be the goal of surgery since the safety and efficacy of stereotactic radiosurgery are not guaranteed in every patient. ${ }^{23}$

\section{Evidence in Favor of the Accuracy of the Simpson Grade and Its Modifications}

Several authors have maintained an allegiance to the Simpson grading scale, but when examined closely, the primary purpose of their arguments is to advocate for the goal of striving for maximal safe resection, rather than supporting the grading system itself. A close reading of papers supporting the Simpson grade indicates that while their results clearly show that a GTR reduces recurrence over STR, the data do not actually bolster Simpson's 5-grade scale. These studies generally use the Simpson grade as a surrogate for establishing that extent of resection is an important factor in predicting recurrences. However, proving that extent of resection reduces the risk of recurrences is not the same as claiming that Simpson's 5-grade scale is an ideal or even useful predictor of recurrence.

\section{Skull Base Tumors and the Modified Shinso or Okudesa- Kobayashi Grade}

DeMonte et al. attempted to update the Simpson scale in a series of cavernous sinus meningiomas by creating a scale from unpublished data of Kobayashi et al. (Table 3). ${ }^{24}$ The weakness of this scale is that these new subdivisions are not based on outcome data, but rather the surgeon's impression of what can be accomplished intraoperatively, particularly during removal of skull base meningiomas. The authors created a grade for tumor left on a cranial 
TABLE 3. Modified tumor removal grading system of Kobayashi et al.*

\begin{tabular}{cc}
\hline Grade & Definition \\
\hline I & $\begin{array}{c}\text { Complete microscopic removal of tumor and dural attachment } \\
\text { with any abnormal bone }\end{array}$ \\
\hline II & $\begin{array}{c}\text { Complete microscopic removal of tumor with diathermy } \\
\text { coagulation of its dural attachment }\end{array}$ \\
\hline III & $\begin{array}{c}\text { Complete microscopic removal of intra- and extradural tumor } \\
\text { without resection or coagulation of its dural attachment }\end{array}$ \\
\hline III & $\begin{array}{c}\text { Complete microscopic removal of intradural tumor without } \\
\text { resection or coagulation of its dural attachment or of any } \\
\text { extradural extensions }\end{array}$ \\
\hline IV $V_{A}$ & $\begin{array}{c}\text { Intentional subtotal removal to preserve cranial nerves or } \\
\text { blood vessels with complete microscopic removal of } \\
\end{array}$ \\
attachment
\end{tabular}

Reproduced from DeMonte F, Smith HK, al-Mefty O. J Neurosurg. 1994;81(2): 245-251.24 Published with permission.

* Also known as the Modified Kobayashi grade/Shinso grade or OkudesaKobayashi grade.

nerve as well as a cutoff of $10 \%$ residual volume, but there are no data that tumor on a cranial nerve is more or less likely to regrow compared with a $<10 \%$ residual volume. The authors claimed, "Kobayashi and colleagues reported a $0 \%$ recurrence rate in 63 patients who had Grade I to III removal of skull-based meningiomas" but failed to see that an equal lack of recurrences for all three grades undermines the need for a distinction between them. In another series of petroclival meningiomas, Natarajan et al. attempted to use a Simpson grading scale but ultimately resorted to classifying their tumors as GTR, STR $(<10 \%$ remaining), or partial resection ( $>10 \%$ remaining). The latter two categories were lumped together in their analysis of progression, further undermining the significance of the $10 \%$ residual threshold. ${ }^{25}$ Similarly, Almefty et al. examined the prognostic significance of the same scale, which they referred to as the Modified Shinso grade or OkudesaKobayashi grade (Table 2$)^{26}$ in a series of petroclival meningiomas. The authors again showed that GTR provides higher rates of tumor control compared with STR, but they did not report recurrences for each of the Kobayashi grades, so the scale's predictive value is unclear other than grade I, which they reported had no recurrences.

\section{Confusing the Simpson Grade With Maximal Safe Resection}

Proponents of the Simpson scale, facing criticism regarding the safety and predictive value of pursing a Simpson grade I result for skull base meningiomas, relied on convexity meningiomas to demonstrate the advantages of complete resection. One such publication by Hasseleid et al. titled "Surgery for convexity meningioma: Simpson Grade I resection as the goal," reported that Simpson grade was predictive of recurrence. ${ }^{27}$ However, for the sake of statistical analysis, Simpson grades II and III were combined, as were grades IV and V, and significance was only achieved when grade I was compared with the other grades. Moreover, grade 0 resection was not attempted, and the 5- and 10-year recurrence rates were only $2.3 \%$ and $4.3 \%$, respectively. This paper only serves to further undermine the reliability of the Simpson grade since the conclusion was that the only relevant grade is grade I, and its application only applies to one location, namely, the convexity.

Heald et al. attempted to defend Simpson's grades but was only able to find statistical significance when tumors were divided into GTR and STR. ${ }^{28}$ Similarly, Gallagher et al. tried to correlate Simpson grade with progression/ recurrence-free survival, but their results showed that the correlation was primarily driven by the distinction between GTR (grades I, II, and III) and STR (grades IV and V). ${ }^{29}$ Likewise, in another study, Nanda et al. needed to combine Simpson grades I and II for comparison with grades III and IV to show significance..$^{30}$

Two of the more compelling studies supporting the Simpson grade can be found in more recent publications by van Alkemade et al..$^{31}$ and Gousias et al. ${ }^{32}$ Both papers showed a graded decrease in recurrence that appeared to conform with Simpson's classification. ${ }^{31,32}$ However, the Simpson grading was obtained by retrospective review of the operative notes as well as the postoperative MRI reports. Not only are surgeons' intraoperative assessments notoriously inaccurate at describing their actual accomplishments, but unless the surgeon provides a Simpson grade in the operative note itself, the conversion of a surgical description into a Simpson grade may be erroneous.

\section{Biological Basis of the Simpson Grade}

Meningiomas can be compartmentalized into the primary mass, adjacent satellite lesions, the dural tail, and regional hyperostotic bone. As discussed above, Borovich and Doron showed that the primary mass is surrounded in roughly $60 \%$ of cases by micro- and macroscopic meningotheliomatous dural nodules as far as $4 \mathrm{~cm}$ from the tumor margin. ${ }^{8}$ This phenomenon is not the same as the dural tail, seen on gadolinium-enhanced MRI scans (Fig. 1). Tokumaru et al. in 1990 first examined the dural tail and found that the majority of the signal arose from loose connective tissue, hypervascularity, and dilated vessels, with tumor cells found only $1 \mathrm{~mm}$ from the tumor margin. ${ }^{33}$ Several other authors have examined the dural tail and found tumor cells in anywhere from $55 \%$ to $88.3 \%$ of cases. $^{34,35}$

Simpson put a great deal of emphasis on removal of adjacent hyperostotic bone to minimize recurrence. However, this assumes that all hyperostotic bone is infiltrated with tumor and that these tumor cells have a propensity to regrow at a rate equivalent to soft tumor. Meningioma-associated hyperostosis was described in detail by Cushing in 1922, who reported hyperostosis in $25 \%$ of cases almost universally caused by tumor invasion..$^{36}$ The pathophysiology of hyperostosis is unknown, and, besides direct tumor invasion, ${ }^{37}$ other possibilities include the release of "humoral factors," which would imply that tumor invasion is not required for hyperostosis. ${ }^{38,39}$ While some authors 
TABLE 4. MEningioma Group Amsterdam Grading System of Meningioma Removal Based on Postoperative Magnetic Resonance Imaging*

\begin{tabular}{cc}
\hline Grade & \multicolumn{1}{c}{ Extent of Resection Based on Postoperative MRI } \\
\hline 1 & $\begin{array}{c}\text { Radiologically complete removal of tumor with excision of its } \\
\text { dural attachment and of any abnormal bone }\end{array}$ \\
\hline 2 & $\begin{array}{c}\text { Radiologically complete removal of the intradural tumor with- } \\
\text { out resection of its dural attachments (e.g., invaded sinus, } \\
\text { hyperostotic bone) }\end{array}$ \\
\hline 3 & Partial removal leaving tumor in situ \\
\hline 4 & Simple decompression \\
\hline
\end{tabular}

Reprinted from World Neurosurg, vol 111, Slot KM, Verbaan D, Bosscher L, et al., "Agreement between extent of meningioma resection based on surgical Simpson grade and based on postoperative magnetic resonance imaging findings," pp e856-e862, 2018, with permission from Elsevier. ${ }^{43}$

* Abbreviated as MEGA.

find tumor invasion in the majority of cases, ${ }^{40}$ others report much lower rates or rates that vary with locations. ${ }^{41}$

The inconsistent rate of tumor invasion into the dural tail and anatomical variability in tumor invasion into adjacent hyperostosis raises the question of whether meningiomas in different locations represent distinct histopathological and genetic subtypes with a different propensity to invade, grow, and transform. The strongest evidence for meningioma diversity based on location can be found in the recent work from Günel's team..$^{42}$ In a series of publications, his laboratory has demonstrated a variety of somatic coding mutations that vary based on the site of origin and confer to tumors arising in each cranial compartment its own unique characteristics. The diversity of meningioma biology and activity undermines any attempt to create a one-size-fits-all treatment strategy based on a unified resection scale.

\section{Summary of the Literature}

In 1957, it was unclear if meningiomas could be cured with surgery and how to achieve that goal. In that context, Simpson introduced his seminal grading scale to motivate surgeons to try to remove as much tumor, involved dura, and bone as possible, since there were no other treatment options available at that time. It is remarkable that after more than 60 years, the Simpson scale is still used in many publications as the gold standard for meningioma surgery, in spite of the numerous revolutions in surgical technique, imaging, molecular biology, and stereotactic radiotherapy (Table 2).

Unfortunately, the Simpson grade has become a surrogate for aggressive surgery, and its supporters wave it like a flag in a war against what they see as substandard, inadequate operations. Simpson himself supported the primary goal of advocating for "maximal safe resection" and offered his scale as a suggestion. In the modern era, the threshold for "safe" is a moving target. The amount of morbidity one is willing to accept to remove more tumor depends on the safety and efficacy of the available alternative treatment should the tumor recur. Adherence to this one-size-fits-all dogma can be dangerous to patient outcomes. That being said, the surgeon should try to do everything in his or her power to achieve as aggressive a resection as possible, which should include removal and cauterization of adjacent dura and invaded bone, but only if this can be achieved with minimal morbidity. Given that the size of the bone opening can limit the surgeon's ability to inspect for lateral dural remnants, the only objective, reliable, and widely available measure of surgical success is radiographic GTR (Fig. 1).

\section{Future Directions}

In a recent article, Slot et al. introduced a modified meningioma resection scale that relies on postoperative MRI findings, which they call the MEGA (Meningioma Group Amsterdam Grading System of Meningioma Removal Based on Postoperative Magnetic Resonance Imaging) scale (Table 4) ${ }^{43}$ Currently, their scale has not been correlated with recurrences. Although it suffers from some of the same issues as the Simpson grade (e.g., assumptions about hyperostotic bone, no definition of degree of partial resection), it may be a move in the right direction. Future studies using radiomics and artificial intelligence algorithms, as well as volumetric postoperative MRI surveillance should be collected in large numbers to answer questions about surrogate imaging markers for biology and risk of recurrence. Evidence is needed to quantify the risk of regrowth of hyperostotic bone and determine if partial removal of involved bone reduces recurrence. Similarly, the volume of residual meningioma and its regrowth potential should be investigated in greater detail. These studies must separate tumors based on location, since genetics and WHO grade cannot reliably be determined preoperatively.

Ultimately, pre-, post-, and intraoperative imaging will be guided by advances in imaging and molecularly tagged radioactive or fluorescent markers. Somatostatin-receptor 2A (SSTR2A) is a highly sensitive and specific marker for meningiomas, ${ }^{44}$ and [68Ga]-DOTATATE-PET provides more accurate imaging of meningioma invasion than does MRI. ${ }^{45}$ Routine [68Ga]-DOTATATE-PET imaging of meningiomas will help surgeons identify tumor cells in the dural tail and hyperostotic bone and help guide the goals of surgery, rather than relying on a single scale (Fig. 1) ${ }^{46}$ Intraoperative fluorescent markers such as 5-aminolevulinic acid and fluorescein have already been shown to be useful at demonstrating meningioma cells, including invaded hyperostotic bone. ${ }^{47,48}$ Ideally, an SSTR2A fluorescent marker will be employed to increase the sensitivity and specificity of intraoperative fluorescence-guided surgery.

\section{Conclusions}

Donald Simpson's name and his scale deserve to be recognized and preserved in the historical pantheon of pioneering and transformative neurosurgical concepts. Nevertheless, his eponymous scale seems no longer relevant in modern meningioma surgery. While his message of achieving maximal safe tumor removal is still germane, a single measure using subjective criteria cannot be applied universally to all meningioma surgeries, regardless of location. Objective measures of extent of resection using MRI, and eventually targeted molecular markers, must be employed to determine surgical goals and outcomes. 


\section{References}

1. Simpson D. The recurrence of intracranial meningiomas after surgical treatment. J Neurol Neurosurg Psychiatry. 1957; 20(1):22-39.

2. Louis DN, Ohgaki H, Wiestler OD, et al. The 2007 WHO classification of tumours of the central nervous system. Acta Neuropathol. 2007;114(2):97-109.

3. Cushing H, Eisenhardt L. Meningiomas: Their Classification, Regional Behaviour, Life History, and Surgical End Results. Charles C. Thomas; 1938.

4. Adegbite AB, Khan MI, Paine KWE, Tan LK. The recurrence of intracranial meningiomas after surgical treatment. $J$ Neurosurg. 1983;58(1):51-56.

5. Mirimanoff RO, Dosoretz DE, Linggood RM, et al. Meningioma: analysis of recurrence and progression following neurosurgical resection. J Neurosurg. 1985;62(1): $18-24$.

6. Naumann M, Meixensberger J. Factors influencing meningioma recurrence rate. Acta Neurochir (Wien). 1990;107(3-4): $108-111$.

7. Jääskeläinen J. Seemingly complete removal of histologically benign intracranial meningioma: late recurrence rate and factors predicting recurrence in 657 patients. A multivariate analysis. Surg Neurol. 1986;26(5):461-469.

8. Borovich B, Doron Y. Recurrence of intracranial meningiomas: the role played by regional multicentricity. J Neurosurg. 1986;64(1):58-63.

9. Maiuri F, Mariniello G, Peca C, et al. Multicentric and diffuse recurrences of meningiomas. Br J Neurosurg. Published online April 21, 2020. doi:10.1080/02688697.2020.1754335

10. Borovich B, Doron Y, Braun J, et al. Recurrence of intracranial meningiomas: the role played by regional multicentricity. Part 2: Clinical and radiological aspects. J Neurosurg. 1986; 65(2):168-171.

11. Kinjo T, al-Mefty O, Kanaan I. Grade zero removal of supratentorial convexity meningiomas. Neurosurgery. 1993;33(3): 394-399.

12. Morokoff AP, Zauberman J, Black PM. Surgery for convexity meningiomas. Neurosurgery. 2008;63(3):427-434.

13. McGovern SL, Aldape KD, Munsell MF, et al. A comparison of World Health Organization tumor grades at recurrence in patients with non-skull base and skull base meningiomas. $J$ Neurosurg. 2010;112(5):925-933.

14. Sughrue ME, Kane AJ, Shangari G, et al. The relevance of Simpson Grade I and II resection in modern neurosurgical treatment of World Health Organization Grade I meningiomas. J Neurosurg. 2010;113(5):1029-1035.

15. Oya S, Kawai K, Nakatomi H, Saito N. Significance of Simpson grading system in modern meningioma surgery: integration of the grade with MIB-1 labeling index as a key to predict the recurrence of WHO Grade I meningiomas. $J$ Neurosurg. 2012;117(1):121-128.

16. Otero-Rodriguez A, Tabernero MD, Munoz-Martin MC, et al. Re-evaluating Simpson Grade I, II, and III resections in neurosurgical treatment of World Health Organization grade I meningiomas. World Neurosurg. 2016;96:483-488.

17. Ehresman JS, Garzon-Muvdi T, Rogers D, et al. The relevance of Simpson grade resections in modern neurosurgical treatment of World Health Organization grade I, II, and III meningiomas. World Neurosurg. 2018;109:e588-e593.

18. Voß KM, Spille DC, Sauerland C, et al. The Simpson grading in meningioma surgery: does the tumor location influence the prognostic value? J Neurooncol. 2017;133(3):641-651.

19. Fukushima Y, Oya S, Nakatomi H, et al. Effect of dural detachment on long-term tumor control for meningiomas treated using Simpson grade IV resection. J Neurosurg. 2013; 119(6):1373-1379.

20. Materi J, Mampre D, Ehresman J, et al. Predictors of recurrence and high growth rate of residual meningiomas after subtotal resection. J Neurosurg. Published online January 3, 2020. doi:10.3171/2019.10.JNS192466

21. Black PM, Villavicencio AT, Rhouddou C, Loeffler JS. Aggressive surgery and focal radiation in the management of meningiomas of the skull base: preservation of function with maintenance of local control. Acta Neurochir (Wien). 2001; 143(6):555-562.

22. Condra KS, Buatti JM, Mendenhall WM, et al. Benign meningiomas: primary treatment selection affects survival. Int $J$ Radiat Oncol Biol Phys. 1997;39(2):427-436.

23. Rogers L, Mehta M. Role of radiation therapy in treating intracranial meningiomas. Neurosurg Focus. 2007;23(4):E4.

24. DeMonte F, Smith HK, al-Mefty O. Outcome of aggressive removal of cavernous sinus meningiomas. J Neurosurg. 1994; 81(2):245-251.

25. Natarajan SK, Sekhar LN, Schessel D, Morita A. Petroclival meningiomas: multimodality treatment and outcomes at long-term follow-up. Neurosurgery. 2007;60(6):965-981.

26. Almefty R, Dunn IF, Pravdenkova S, et al. True petroclival meningiomas: results of surgical management. J Neurosurg. 2014;120(1):40-51.

27. Hasseleid BF, Meling TR, Rønning P, et al. Surgery for convexity meningioma: Simpson Grade I resection as the goal: clinical article. J Neurosurg. 2012;117(6):999-1006.

28. Heald JB, Carroll TA, Mair RJ. Simpson grade: an opportunity to reassess the need for complete resection of meningiomas. Acta Neurochir (Wien). 2014;156(2):383-388.

29. Gallagher MJ, Jenkinson MD, Brodbelt AR, et al. WHO grade 1 meningioma recurrence: are location and Simpson grade still relevant? Clin Neurol Neurosurg. 2016;141:117121.

30. Nanda A, Bir SC, Maiti TK, et al. Relevance of Simpson grading system and recurrence-free survival after surgery for World Health Organization Grade I meningioma. J Neurosurg. 2017;126(1):201-211.

31. van Alkemade H, de Leau M, Dieleman EMT, et al. Impaired survival and long-term neurological problems in benign meningioma. Neuro Oncol. 2012;14(5):658-666.

32. Gousias K, Schramm J, Simon M. The Simpson grading revisited: aggressive surgery and its place in modern meningioma management. J Neurosurg. 2016;125(3):551-560.

33. Tokumaru A, O'uchi T, Eguchi T, et al. Prominent meningeal enhancement adjacent to meningioma on Gd-DTPA-enhanced MR images: histopathologic correlation. Radiology. 1990;175(2):431-433.

34. Qi ST, Liu Y, Pan J, et al. A radiopathological classification of dural tail sign of meningiomas. J Neurosurg. 2012;117(4): 645-653.

35. Slot KM, Verbaan D, Uitdehaag BMJ, et al. Can excision of meningiomas be limited to resection of tumor and radiologically abnormal dura mater? Neuronavigation-guided biopsies of dural tail and seemingly normal dura mater, with a review of the literature. World Neurosurg. 2014;82(6):e832-e836.

36. Cushing $\mathrm{H}$. The cranial hyperostoses produced by meningeal endotheliomas. Arch Neurol Psychiatry. 1922;8(2):139-154.

37. Echlin F. Cranial osteomas and hyperostoses produced by meningeal fibroblastomas. A clinical pathologic study. Arch Surg. 1934;28(2):357-405.

38. Heick A, Mosdal C, Jørgensen K, Klinken L. Localized cranial hyperostosis of meningiomas: a result of neoplastic enzymatic activity? Acta Neurol Scand. 1993;87(3):243-247.

39. Di Cristofori A, Del Bene M, Locatelli M, et al. Meningioma and bone hyperostosis: expression of bone stimulating factors and review of the literature. World Neurosurg. 2018;115: e774-e781.

40. Pieper DR, Al-Mefty O, Hanada Y, Buechner D. Hyperostosis associated with meningioma of the cranial base: secondary changes or tumor invasion. Neurosurgery. 1999;44(4): $742-747$. 
41. Lau BL, Che Othman MI, Fathil MFMD, et al. Does putting back hyperostotic bone flap in meningioma surgery cause tumor recurrence? An observational prospective study. World Neurosurg. 2019;127:e497-e502.

42. Youngblood MW, Duran D, Montejo JD, et al. Correlations between genomic subgroup and clinical features in a cohort of more than 3000 meningiomas. J Neurosurg. 2020;133(5):1345-1354.

43. Slot KM, Verbaan D, Bosscher L, et al. Agreement between extent of meningioma resection based on surgical Simpson grade and based on postoperative magnetic resonance imaging findings. World Neurosurg. 2018;111:e856-e862.

44. Menke JR, Raleigh DR, Gown AM, et al. Somatostatin receptor $2 \mathrm{a}$ is a more sensitive diagnostic marker of meningioma than epithelial membrane antigen. Acta Neuropathol. 2015;130(3):441-443.

45. Ivanidze J, Roytman M, Lin E, et al. Gallium-68 DOTATATE PET in the evaluation of intracranial meningiomas. $J$ Neuroimaging. 2019;29(5):650-656.

46. Kunz WG, Jungblut LM, Kazmierczak PM, et al. Improved detection of transosseous meningiomas using ${ }^{68} \mathrm{Ga}$-DOTATATE PET/CT compared with contrast-enhanced MRI. $J$ Nucl Med. 2017;58(10):1580-1587.

47. Valdes PA, Millesi M, Widhalm G, Roberts DW. 5-aminolevulinic acid induced protoporphyrin IX (ALA-PpIX) fluorescence guidance in meningioma surgery. J Neurooncol. 2019; 141(3):555-565.
48. Akçakaya MO, Göker B, Kasımcan MÖ, et al. Use of sodium fluorescein in meningioma surgery performed under the YELLOW-560 nm surgical microscope filter: feasibility and preliminary results. World Neurosurg. 2017;107:966-973.

49. Grant FC. A clinical experience with meningiomas of the brain. J Neurosurg. 1954;11(5):479-487.

\section{Disclosures}

The authors report no conflict of interest concerning the materials or methods used in this study or the findings specified in this paper.

\section{Author Contributions}

Conception and design: both authors. Drafting the article: Schwartz. Critically revising the article: both authors. Reviewed submitted version of manuscript: Schwartz. Approved the final version of the manuscript on behalf of both authors: Schwartz.

\section{Correspondence}

Theodore H. Schwartz: Weill Cornell Medicine, NewYork-Presbyterian Hospital, New York, NY. schwarh@med.cornell.edu. 